\title{
A Search for New Emission Nebulae from the SHASSA and VTSS Surveys
}

\author{
David J. Frew ${ }^{1}$, G.J. Madsen ${ }^{2}$ and Q.A. Parker ${ }^{1,2}$ \\ ${ }^{1}$ Department of Physics, Macquarie University, NSW 2109, Australia, \\ email: dfrew@ics.mq.edu.au \\ ${ }^{2}$ Anglo-Australian Observatory, PO Box 296, Epping, NSW 1710, Australia
}

\begin{abstract}
As an adjunct to the planetary nebula (PN) search from the AAO/UKST H $\alpha$ survey, a visual search was conducted for new emission nebulae from the SHASSA and VTSS surveys, outside a Galactic latitude of $|b|=10^{\circ}$. Fifteen new objects were found from SHASSA and three from the available fields of VTSS. With one exception, all objects are $>5^{\prime}$ across, as smaller nebulae are confused with large numbers of artifacts and compact emitters on these surveys. All previously known PNe larger than this size in the search area, as well as Hewett 1, PG 0108, and PG 0109 were recovered in this blind search. Candidates were selected as discrete, morphologically symmetric $\mathrm{H} \alpha$ enhancements, to differentiate them from the ubiquitous diffuse emission structure of the ISM. These criteria were relaxed for the VTSS survey due to its poorer inherent resolution. Most of the new discoveries are probable Stromgren spheres in the ISM. Some show unusual line ratios (e.g. strong [O III] or [N II] emission) based on slit spectroscopy and WHAM data (see Madsen et al. 2006, this volume), suggesting these are ionised by a hot subdwarf or white dwarf star, and may be possible PNe. Our most interesting discovery is a rare bowshock nebula around a bright, previously unnoticed, nova-like cataclysmic variable.
\end{abstract}

Keywords. (ISM:) planetary nebulae: general, (ISM:) HII regions

\section{Introduction}

Large evolved planetary nebulae $(\mathrm{PNe})$ dominate any volume-limited PN census. Numerous highly evolved PN candidates have been discovered from the AAO/UKST H $\alpha$ Survey (see Parker et al. 2005), a high resolution $\mathrm{H} \alpha+[\mathrm{N}$ II] survey of the southern Galactic plane using the 1.2-m UKST. This survey has led to the discovery of $~ 900$ new Galactic PNe, presented as the Macquarie/AAO/Strasbourg/H $\alpha$ (MASH) Catalogue (Parker et al. 2006).

As an adjunct to this survey, the first author has utilised the Southern H $\alpha$ Sky Survey Atlas (SHASSA; Gaustad et al. 2001) and the Virginia Tech Spectral line Survey (VTSS; Dennison, Simonetti \& Topasna 1998) to search for emission enhancements outside the zone covered by the AAO/UKST $\mathrm{H} \alpha$ survey, i.e. above $|b|=10^{\circ}$. Both of these CCD surveys used fast short-focus lenses, so have modest angular resolution; $48^{\prime \prime} \mathrm{pixel}^{-1}$ for SHASSA and $96^{\prime \prime}$ pixel $^{-1}$ for VTSS. Despite this poor resolution, both surveys are very deep (down to $\sim 1$ Rayleigh at $\mathrm{H} \alpha$ ) and are a useful tool to search for any large evolved high-latitude PNe that may have been missed previously.

\section{Method and Results}

Both surveys, but especially SHASSA, contain large numbers of artifacts, compact emitters and variable stars. This precludes the use of an automated search technique for new PN candidates. Hence a visual search was conducted of all SHASSA fields plus the VTSS fields that are currently available online. Nebula candidates were selected 
Table 1. H $\alpha$ nebulae discovered from SHASSA and VTSS surveys. The positions are for J2000. Column 7 gives the field number (SHASSA fields have the 3 digit code), while columns 8 and 9 give the ID and $V$ magnitude of the suggested ionizing star candidate. The last column gives alternative identifications for the nebulae, including objects from the WHAM Point Source Catalogue (WPS; Reynolds et al. 2005).

\begin{tabular}{|c|c|c|c|c|c|c|c|c|c|c|}
\hline Name & R.A. & Dec. & $l$ & $b$ & $\begin{array}{c}\text { Dimensions } \\
\text { (arcmin) }\end{array}$ & Field & Star & $V$ & Type & Other ID \\
\hline Fr $2-1$ & 010200 & -611800 & 00.9 & -55.8 & $100 \times 100$ & 025 & LB 3174 & 12.34 & HII? & \\
\hline Fr $2-2$ & 024046 & $\begin{array}{lll}-10 & 21 & 16\end{array}$ & 161.9 & -44.1 & $15 \times 15$ & 238 & & & $?$ & WPS 53 \\
\hline Fr $2-3$ & 045620 & -280748 & 229.3 & -36.2 & $20 \times 15$ & 238 & RE $0457-28$ & 13.95 & HII? & 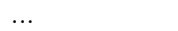 \\
\hline Fr 2-4 & $07 \quad 1152$ & -820303 & 294.1 & -26.1 & $90 \times 60$ & 004 & $\ldots$ & $\ldots$ & HII? & . \\
\hline Fr $2-5$ & $\begin{array}{llll}08 & 10 & 14\end{array}$ & -672722 & 280.7 & -17.8 & $80 \times 45$ & 01 & $\ldots$ & $\ldots$ & HII? & \\
\hline Fr 2-6 & 084023 & -575449 & 274.3 & -9.8 & $8.2 \times 6.3$ & 0 & & & PN? & FP \\
\hline Fr 2-7 & 105410 & -701145 & 293.2 & -9.6 & $8.0 \times 7.5$ & 01 &. & . & PN? & FP 1054-7011 \\
\hline Fr $2-8$ & 140043 & $\begin{array}{lll}-51 & 02 & 12\end{array}$ & 313.9 & +10.4 & $2.0 \times 1.9$ & 05 & ... & $\ldots$ & $\mathrm{PN}$ & AM $1357-504$ \\
\hline Fr 2-9 & 142331 & -091848 & 337.6 & +47.3 & $80 \times 60$ & 18 & G124- & 15.48 & HII? & WPS 82 \\
\hline Fr $2-10$ & $\begin{array}{lll}15 & 09 & 19\end{array}$ & -052054 & 353.9 & +43.5 & $30 \times 30$ & 720 & PG 1506-052 & 14.0 & HII? & $\ldots$ \\
\hline Fr $2-11$ & 165748 & $\begin{array}{lll}-63 & 12 & 00\end{array}$ & 327.5 & -12.5 & $8.0 \times 6.0$ & 037 & V341 Ara & $10.7_{v}$ & $\mathrm{CV}$ & 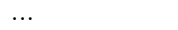 \\
\hline Fr $2-12$ & $\begin{array}{lll}17 & 21 & 09\end{array}$ & -565425 & 333.9 & -11.3 & $7.3 \times 6.0$ & 038 & $\ldots$ & $\ldots$ & $\mathrm{PN}$ & FP 1721-5654 \\
\hline Fr $2-13$ & 175900 & +342936 & 60.5 & +25.1 & $15 \times 12$ & Her13 & .. & $\ldots$ & HII? & \\
\hline Fr $2-14$ & 202600 & +763700 & 109.8 & +21.1 & $90 \times 80$ & Cep08 & .. & $\ldots$ & HII? & WH \\
\hline Fr $2-15$ & 202716 & +114920 & 55.1 & -15.1 & $25 \times 20$ & 265 & $\ldots$ & $\ldots$ & HII? & WPS 16 \\
\hline Fr $2-16$ & 211830 & +120136 & 62.9 & -25.1 & $30 \times 15$ & 266 & HS2115+1148 & 16.5 & HII? & $\ldots$ \\
\hline Fr $2-17$ & 211945 & -562345 & 339.7 & -42.7 & $80 \times 30$ & 041 & $\ldots$ & $\ldots$ & HII? & $\ldots$ \\
\hline Fr $2-18$ & 231141 & +292754 & 98.2 & -28.6 & $30 \times 25$ & Peg13 & $\cdots$ & $\ldots$ & HII? & $\ldots$ \\
\hline
\end{tabular}

as discrete, symmetric $\mathrm{H} \alpha$ enhancements, to differentiate them from the diffuse ISM background. Fifteen nebulae were found from SHASSA and three from VTSS. With one exception, all objects are $>5^{\prime}$ across. All previously known PNe larger than this size, as well as PHL 932, Hewett 1, PG 0108+101 and PG 0109+111 were recovered in this blind search. Table 1 summarizes the main properties of the new nebulae.

Most of the new discoveries are probable Strömgren spheres in the ISM. However, some show unusual line ratios (e.g. strong [O III] or [N II] emission) based on long-slit spectroscopy or observations with the Wisconsin $\mathrm{H} \alpha$ Mapper (WHAM; Haffner et al. 2003), which suggest these nebulae are ionized by a very hot subdwarf or white dwarf star, and may be possible PNe. The ability of WHAM to provide velocity information has allowed us to determine the true nature of several of these objects (Madsen et al. 2006, this conference). A follow-up program of imagery and spectroscopy is underway to determine the nature of the nebulae too far south for WHAM. Our most interesting discovery is a rare bowshock nebula around a bright, previously unnoticed, nova-like cataclysmic variable, V341 Ara.

\section{References}

Dennison, B., Simonetti, J.H., \& Topasna, G.A. 1998, PASA 15, 147

Gaustad, J.E., McCullough, P.R., Rosing, W., \& Van Buren, D. 2001, PASP 113, 1326

Haffner, L.M., Reynolds, R.J., Tufte, S.L., Madsen, G.J., Jaehnig, K.P., \& Percival, J.W. 2003, ApJS 149, 405

Parker, Q.A., Phillipps, S., \& Pierce, M.J., et al. 2005, MNRAS 362, 689

Parker, Q.A., Acker, A., Frew, D.J., et al. 2006, MNRAS in press

Reynolds, R.J., Chaudhary, V., Madsen, G.J., \& Haffner, L.M. 2005, AJ 129, 927 